\title{
ATIVIDADE MICROBIANA, CARBONO E NITROGÊNIO DA BIOMASSA MICROBIANA EM PLANTAÇÕES DE EUCALIPTO, EM SEQÜÊNCIA DE IDADES ${ }^{(1)}$
}

\author{
Patrícia Anjos Bittencourt Barreto ${ }^{(2)}$, Emanuela Forestieri da \\ Gama-Rodrigues $^{(3)}$, Antonio Carlos da Gama-Rodrigues ${ }^{(3)}$, \\ Nairam Félix de Barros ${ }^{(4)} \&$ Sebastião Fonseca ${ }^{(5)}$
}

\begin{abstract}
RESUMO
Em plantações florestais, a atividade microbiana tem grande relevância para a ciclagem de nutrientes e a fertilidade do solo, uma vez que esses ecossistemas, devido a seus característicos longos períodos de rotação, proporcionam um contínuo aporte de serapilheira e morte de raízes, que contribuem para a manutenção e elevação do teor de matéria orgânica do solo. O objetivo deste trabalho foi caracterizar a atividade e a biomassa microbiana em solos sob plantações de eucalipto em distintas idades de cultivo. Foram avaliadas alterações na biomassa e atividade microbiana em solo e serapilheira de plantações de eucalipto com 1, 3, 5 e 13 anos de idade crescendo sob condições edafoclimáticas semelhantes. Encontraram-se maiores teores de $\mathrm{C}$ e $\mathrm{N}$ microbiano da serapilheira do que no solo, sugerindo que a serapilheira seria uma importante reserva de $\mathrm{C}$ e $\mathrm{N}$ microbiano em povoamentos de eucalipto. Não se verificou tendência de aumento ou diminuição da biomassa e da atividade microbiana com as idades das plantações, o que, provavelmente, decorreu do fato dos povoamentos estudados corresponderem a áreas de reforma. A qualidade orgânica da serapilheira influenciou diretamente a atividade, o $\mathrm{C}$ e $\mathrm{N}$ da biomassa microbiana da serapilheira. Os indicadores mais sensíveis para aferir variações dos solos das plantações de eucalipto foram os teores de C orgânico e $\mathrm{N}$ total, e na serapilheira foram os atributos microbianos, os teores de celulose, lignina $\mathrm{e}$ N.
\end{abstract}

Termos de indexação: matéria orgânica, ciclagem de nutrientes, solos florestais.

\footnotetext{
(1) Parte da Tese de Mestrado da primeira autora, apresentada à Universidade Estadual do Norte Fluminense - UENF. Recebido para publicação em julho de 2007 e aprovado em outubro de 2007.

${ }^{(2)}$ Doutorando da Universidade Estadual Norte Fluminense - UENF/CCTA. Laboratório de Solos, CEP 28013-602 Campos dos Goytacazes (RJ). Bolsista da UENF-FAPERJ. E-mail: barretopab@hotmail.com

(3) Professor Associado do Laboratório de Solos, CCTA/UENF. E-mails: emanuela@uenf.br; tonygama@uenf.br

(4) Professor Titular do Departamento de Solos, Universidade Federal de Viçosa - UFV. CEP 36570-000 Viçosa (MG). Bolsista do CNPq. E-mail: nfbarros@mail.ufv.br

(5) Engenheiro Florestal, Pesquisador da Aracruz Celulose S.A. Caixa Postal 331011, CEP 29197-000 Aracruz (ES). E-mail: sf@aracruz.com.br
} 


\title{
SUMMARY: ACTIVITY, CARBONAND NITROGENOF MICROBIAL BIOMASS IN EUCALYPT PLANTATIONS IN AN AGE SEQUENCE
}

\begin{abstract}
Microbial activity is highly relevant for nutrient cycling and soil fertility in forest plantations. Due to the long growth cycles, continuous litter production and dead roots contribute to storage and increase of soil organic matter. Changes in soil and litter microbial biomass and activity under 1, 3, 5 and 13-year-old eucalypt plantations were evaluated. Soil and climate conditions were the same at the sites. Microbial biomass $C$ and $N$ were higher in litter than in soil. Then, litter could be an important sink of microbial $C$ and $N$ in eucalypt plantation. No increase or drop in microbial biomass and activity was observed as plantations grew older, probably because the soil and litter samples were from reformed plantations. Litter quality had a direct influence on litter microbial activity and microbial biomass $C$ and $N$. Organic carbon and $N$ content were the most reliable indicators to show changes in the soil under eucalypt plantations, while in the litter it were the microbial attributes and cellulose, lignin, and $N$ content.
\end{abstract}

Index terms: organic matter, nutrient cycling, forest soils.

\section{INTRODUÇÃO}

No Brasil, os plantios de eucalipto têm sido realizados em diversos tipos de solos que apresentam teores disponíveis e totais de nutrientes numa faixa bastante ampla (Barros \& Comerford, 2002). Porém, a maior parte desses solos é ácida e apresenta baixa disponibilidade de nutrientes. Assim, para atingir maior produtividade, que requer elevada demanda de nutrientes, há a necessidade de aplicação de altas quantidades de fertilizante (Barros et al., 2004). Dentre os elementos minerais, o N é normalmente absorvido e acumulado em maior quantidade (Poggiani et al.,1983; Reis et al., 1987; Schumacher \& Poggiani, 1993). Contudo, a intensidade de resposta do eucalipto à adubação nitrogenada é de baixa magnitude (Barros et al., 1990; Gonçalves et al., 2000), o que faz crer que as diversas fontes naturais de $\mathrm{N}$ sejam suficientes para atender à demanda das plantas de eucalipto (Gonçalves et al., 2001).

$\mathrm{O}$ ciclo do $\mathrm{N}$ no solo está estreitamente associado à matéria orgânica, por isso a biomassa microbiana tem sido considerada sua importante fonte, uma vez que constitui sua fração ativa e potencialmente mineralizável (Marumoto et al., 1982; Bonde et al., 1988). A ciclagem do N microbiano é mais rápida do que as de outras frações da matéria orgânica do solo (MOS) (Smith \& Paul, 1990). A biomassa microbiana atua como um tampão do $\mathrm{N}$ no solo, controlando a disponibilidade desse nutriente por meio dos processos de mineralização e imobilização (Vargas \& Scholes, 1998; Gama-Rodrigues et al., 2005). Gama-Rodrigues (1997a) observou que, numa seqüência de idades, o fluxo de $\mathrm{N}$ gerado pela ciclagem microbiana em povoamentos de eucalipto é suficiente para suprir $78 \%$ da demanda de cultivo de dois anos e até $51 \%$ da demanda do de sete anos.
A biomassa microbiana representa a fração lábil da MOS, de natureza dinâmica e facilmente alterada por fatores bióticos e abióticos (Powlson et al., 1987; Gama-Rodrigues, 1999). Em razão disso, apesar de representar pequena parte do C orgânico do solo, a biomassa microbiana tem sido considerada um indicador mais sensível de mudanças nos tores de MOS do que o teor de C orgânico do solo (Powlson et al., 1987; Anderson \& Domsch, 1989; Sparling, 1992; Gama-Rodrigues, 1999). Em solos sob diferentes coberturas florestais no sul da Bahia, Gama-Rodrigues (1997b) observou apenas diferença significativa nos teores de $\mathrm{C}$ e $\mathrm{N}$ da biomassa microbiana quando comparados com os teores totais desses elementos no solo. D'Andréa et al. (2002) observaram alterações significativas no $\mathrm{C}$ da biomassa microbiana do solo decorrentes da adoção de sistemas de manejo do solo (plantio direto, plantio convencional e pastagem) em relação ao Cerrado nativo.

Os resultados de $\mathrm{C}$ e $\mathrm{N}$ da biomassa microbiana do solo, quando associados aos resultados da atividade microbiana $\left(\mathrm{CO}_{2}\right.$ liberado) e atributos químicos, como C orgânico e N total, possibilitam a obtenção de índices microbianos que expressam a dinâmica do $\mathrm{C}$ e $\mathrm{N}$ de maneira mais adequada que os valores absolutos (Monteiro \& Gama-Rodrigues, 2004). A relação entre $\mathrm{C}$ da biomassa microbiana e o C orgânico do solo reflete a qualidade da MOS, assim como a eficiência de conversão do $\mathrm{C}$ do solo em $\mathrm{C}$ microbiano. Da mesma maneira, a relação entre $\mathrm{N}$ da biomassa microbiana e o $\mathrm{N}$ total do solo indica a eficiência de conversão do $\mathrm{N}$ do solo em N microbiano (Sparling, 1992). Por sua vez, o quociente metabólico $\left(\mathrm{qCO}_{2}\right)$ (relação entre quantidade de $\mathrm{CO}_{2}$ liberado por unidade de $\mathrm{C}$ da biomassa microbiana e por unidade de tempo), proposto por Anderson \& Domsch (1993), é utilizado para avaliar efeitos ambientais e antropogênicos sobre a atividade 
e a biomassa microbiana. Gama-Rodrigues et al. (1997) verificaram que o $\mathrm{qCO}_{2}$ foi um indicador sensível para estimar o potencial de decomposição da MOS.

Apesar das evidências de que a biomassa microbiana exerce importante papel na dinâmica de C e N em plantações de eucalipto, não há muitos trabalhos relacionados a esse tema no Brasil. Assim, este trabalho teve por objetivo caracterizar a atividade e a biomassa microbiana em solos sob plantações de eucalipto em uma seqüência de idades.

\section{MATERIAL E MÉTODOS}

O estudo foi conduzido no município de AracruzES, em plantações comerciais de eucalipto em uma seqüência de idade (1, 3, 5 e 13 anos), oriundas de um mesmo material genético (clone 1501, Urograndis) e com condições edafoclimáticas semelhantes, relevo plano e solos de textura média a argilosa classificados como Argissolo Amarelo distrófico (Barreto, 2004). O histórico das operações de preparo do solo e de adubação efetuadas nas áreas é apresentado no quadro 1. As coletas de solo e serapilheira foram realizadas em novembro de 2003. Em cada área, estabeleceram-se quatro parcelas (18 x $18 \mathrm{~m}$ ) para amostragem de solo e de serapilheira. Em cada parcela, coletaram-se 20 amostras simples de solo para formar uma composta da camada de $0-10 \mathrm{~cm}$. A coleta de serapilheira foi realizada numa área de $3 \times 3 \mathrm{~m}$ em volta de uma árvore, com DAP (diâmetro altura do peito) médio por parcela.

As amostras dos solos foram analisadas para a determinação do pH em água; $\mathrm{P}$ e $\mathrm{K}$ (extraíveis por Mehlich-1), $\mathrm{Ca}^{2+}, \mathrm{Mg}^{2+}$ e $\mathrm{Al}^{3+}$ (por $\mathrm{KCl} 1 \mathrm{~mol} \mathrm{~L}^{-1}$ ) (Defelipo \& Ribeiro, 1981); C orgânico (oxidação com $\mathrm{K}_{2} \mathrm{Cr}_{2} \mathrm{O}_{7} 1,25 \mathrm{~mol}_{\mathrm{c}} \mathrm{L}^{-1}$ em meio ácido) (Anderson \& Ingram, 1996); $\mathrm{N}$ total (método Kjeldahl) (Embrapa, 1997); e granulometria pelo método da pipeta (Embrapa, 1997) (Quadro 2).

Quadro 1. Histórico do manejo de preparo do solo e adubação das áreas

\begin{tabular}{|c|c|c|c|c|c|}
\hline \multirow{2}{*}{ Plantação ${ }^{(1)}$} & \multirow{2}{*}{$\begin{array}{l}\text { Preparo do } \\
\quad \text { solo }\end{array}$} & \multicolumn{4}{|c|}{ Adubação } \\
\hline & & Pré-plantio & Plantio & Cobertura & Manutenção \\
\hline 1 ano & $\begin{array}{l}\text { Subsolagem } \\
\text { profunda } \\
(90 \mathrm{~cm})\end{array}$ & $\begin{array}{l}\text { - Fosfato natural } \\
\text { reativo } \\
\left(500 \mathrm{~kg} \mathrm{ha}^{-1}\right)\end{array}$ & $\begin{array}{l}\text { - } \\
\text { NPK 06-30-06 + 1,0\% Zn } \\
\left(133 \mathrm{~kg} \mathrm{ha}^{-1}\right)\end{array}$ & & $\begin{array}{l}\text { - Calcário dolomítico } \\
\left(2.500 \mathrm{~kg} \mathrm{ha}^{-1}\right) \\
\text { - NPK } 10-00-30\left(300 \mathrm{~kg} \mathrm{ha}^{-1}\right)\end{array}$ \\
\hline 3 anos & $\begin{array}{l}\text { Subsolagem } \\
(40 \mathrm{~cm})\end{array}$ & $\begin{array}{l}\text { - Fosfato natural } \\
\text { reativo } \\
\left(400 \mathrm{~kg} \mathrm{ha}^{-1}\right)\end{array}$ & $\begin{array}{l}\text { - NPK 06-30-06 + 1,0\% B + } \\
1,0 \% \mathrm{Zn}\left(111 \mathrm{~kg} \mathrm{ha}^{-1}\right)\end{array}$ & $\begin{aligned} \text { - } & \text { NPK 10-00-30 } \\
& \left(111 \mathrm{~kg} \mathrm{ha}^{-1}\right)\end{aligned}$ & $\begin{aligned} & \text { - Calcário dolomítico }\left(2.500 \mathrm{~kg} \mathrm{ha}^{-1}\right) \\
& \text { - } \mathrm{KCl}\left(300 \mathrm{~kg} \mathrm{ha}^{-1}\right) \\
& \text { - } \mathrm{NPK} 10-00-30\left(300 \mathrm{~kg} \mathrm{ha}^{-1}\right)\end{aligned}$ \\
\hline 5 anos & $\begin{array}{l}\text { Coveamento } \\
\text { manual }\end{array}$ & & $\begin{array}{l}\text { - Superfosfato triplo } \\
\left(62 \mathrm{~kg} \mathrm{ha}^{-1}\right)\end{array}$ & & $\begin{aligned} \text { - } & \text { Cinza caldeira de biomassa } \\
& \left(4.000 \mathrm{~kg} \mathrm{ha}^{-1}\right) \\
\text { - } & \text { Superfosfato triplo } \\
& \left(150 \mathrm{~kg} \mathrm{ha}^{-1}\right) \\
\text { - } & \mathrm{KCl}\left(100 \mathrm{~kg} \mathrm{ha}^{-1}\right) \\
\text { - } & \mathrm{NPK} 20-05-20\left(250 \mathrm{~kg} \mathrm{ha}^{-1}\right)\end{aligned}$ \\
\hline 13 anos & $\begin{array}{l}\text { Coveamento } \\
\text { manual }\end{array}$ & & $\begin{aligned} \text { - } & \text { NPK 06-30-06 } \\
& \left(111 \mathrm{~kg} \mathrm{ha}^{-1}\right)\end{aligned}$ & $\begin{array}{l}\text { - Fosfato } \\
\text { acidulado } \\
\left(350 \mathrm{~kg} \mathrm{ha}^{-1}\right)\end{array}$ & - NPK 20-00-20 (160 kg ha $\left.{ }^{-1}\right)$ \\
\hline
\end{tabular}

(1) As plantações foram estabelecidas em 12/2002, 08/2000, 10/1998 e 05/1990, respectivamente.

Quadro 2. Caracterização química e composição granulométrica dos solos sob plantações de eucalipto

\begin{tabular}{|c|c|c|c|c|c|c|c|c|c|c|}
\hline Idade & $\mathbf{p H}_{\mathrm{H}_{2} \mathrm{O}}$ & $\mathbf{P}$ & $\mathrm{Al}^{3+}$ & $\mathbf{K}^{+}$ & $\mathrm{Ca}^{2+}$ & $\mathrm{Mg}^{2+}$ & $\mathbf{H}+\mathbf{A l}$ & Argila & Areia & Silte \\
\hline ano & & $\mathrm{mg} \mathrm{dm}^{-3}$ & 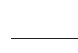 & 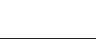 & olc dm & & & & $-\mathrm{g} \mathrm{kg}^{-1}$ & - \\
\hline 1 & $4,8 \mathrm{a}^{(1)}$ & $9,6 \mathrm{~b}$ & $0,5 \mathrm{a}$ & $0,13 \mathrm{a}$ & $1,6 \mathrm{a}$ & $0,6 \mathrm{a}$ & $0,9 b$ & $240,9 \mathrm{a}$ & $718,9 \mathrm{a}$ & $40,2 \mathrm{a}$ \\
\hline 3 & $4,6 \mathrm{a}$ & $6,7 \mathrm{~b}$ & $0,6 \mathrm{a}$ & $0,08 \mathrm{~b}$ & $1,3 \mathrm{ab}$ & $0,6 \mathrm{a}$ & $1,7 \mathrm{a}$ & $212,3 a$ & $755,1 \mathrm{a}$ & $32,5 \mathrm{a}$ \\
\hline 5 & $4,7 \mathrm{a}$ & $19,8 \mathrm{a}$ & $0,5 \mathrm{a}$ & $0,09 \mathrm{~b}$ & $0,6 \mathrm{c}$ & $0,4 \mathrm{a}$ & $0,9 \mathrm{~b}$ & $228,4 a$ & $724,4 \mathrm{a}$ & $47,2 \mathrm{a}$ \\
\hline 13 & $4,5 \mathrm{a}$ & $10,0 \mathrm{~b}$ & $0,8 \mathrm{a}$ & $0,08 \mathrm{~b}$ & $0,9 \mathrm{bc}$ & $0,5 \mathrm{a}$ & $0,9 \mathrm{~b}$ & $243,4 \mathrm{a}$ & $719,4 \mathrm{a}$ & $37,2 \mathrm{a}$ \\
\hline
\end{tabular}

(1) Médias seguidas da mesma letra na coluna não diferem pelo teste de Duncan a $5 \%$. 
As amostras de solo e de serapilheira foram mantidas a $4{ }^{\circ} \mathrm{C}$ (Wardle, 1992), por 10 dias, até execução dos procedimentos de laboratório. As amostras de solo foram homogeneizadas, passadas em peneira $(2 \mathrm{~mm})$ e incubadas por sete dias em recipiente contendo um frasco com água e outro com $\mathrm{NaOH}$ $1 \mathrm{~mol} \mathrm{~L}^{-1}$ para reter o $\mathrm{CO}_{2}$ do solo e eliminar o efeito do peneiramento, o que promove a estabilização das amostras quanto à respiração microbiana. Após esta pré-incubação, as amostras foram ajustadas em suas umidades para $40 \%$ da capacidade máxima de saturação do solo (Grisi, 1995) e, em seguida, procederam-se às análises microbianas.

O método da fumigação-extração foi utilizado para estimar o C (Tate et al., 1988) e o N (Joergensen \& Brookes, 1990) da biomassa microbiana (CBM e NBM, respectivamente) do solo e da serapilheira. A respiração acumulada do solo e a da serapilheira (atividade microbiana) foram estimadas pela quantidade de $\mathrm{CO}_{2}$ liberada (Jenkinson \& Powlson, 1976) em um período de oito e cinco dias de incubação, respectivamente. A incubação foi feita colocando-se cada um dos frascos (snap cap de $150 \mathrm{~mL}$ ) de solo (50 g) e de serapilheira (4 g) em recipientes de $3 \mathrm{~L}$ contendo frascos com $10 \mathrm{~mL}$ de $\mathrm{NaOH} 1 \mathrm{~mol} \mathrm{~L}^{-1}$. Os recipientes foram hermeticamente fechados (com tampa de plástico revestida de borracha) e, após o período de incubação, titulou-se a solução de $\mathrm{NaOH}$ com $\mathrm{HCl}$ $0,5 \mathrm{~mol} \mathrm{~L}^{-1}$, utilizando-se fenolftaleína como indicador.

Determinaram-se os índices microbianos: relações $\mathrm{C}$ da biomassa microbiana/C orgânico (CBM:C), $\mathrm{N}$ da biomassa microbiana/N total (NBM:N) (Sparling, 1992) e o quociente metabólico $\left(\mathrm{qCO}_{2}\right)$, calculado pela relação entre a respiração acumulada e o C da biomassa microbiana (Anderson \& Domsch, 1993), expresso em $\mathrm{mg} \mathrm{CO}_{2} \mathrm{~g}^{-1} \mathrm{dia}^{-1}$ de CBM.

Nas amostras de serapilheira, determinaram-se o teor de $\mathrm{N}$ total pelo método Kjeldahl, descrito por Bataglia et al. (1983) e o teor de C, por oxidação com $\mathrm{K}_{2} \mathrm{Cr}_{2} \mathrm{O}_{7}$ 0,4 $\mathrm{mol} \mathrm{L}^{-1}$ em meio ácido (Anderson \& Ingram, 1996). Além disso, foram realizadas as determinações de polifenóis (polifenóis solúveis, taninos hidrolisáveis e condensados, como também polifenóis não tanínicos) utilizando-se o reagente de Folin-Denis em meio básico, empregando o ácido tânico como padrão e procedendo-se à leitura em espectrofotômetro com comprimento de onda de $760 \mathrm{~nm}$ (Anderson \& Ingram, 1996). Determinaram-se, ainda, os teores de lignina e de celulose pelo método da fibra em detergente ácido (FDA) de Van Soest \& Wine (1968).

Os atributos químicos, microbianos, de qualidade e os índices microbianos do solo e da serapilheira foram analisados segundo um delineamento inteiramente casualizado, com quatro repetições. As médias foram comparadas pelo teste de Duncan a $5 \%$. Os dados também foram submetidos à análise de componentes principais (ACP) com o objetivo de sintetizar sua variação multidimensional em um diagrama, ordenando-os nos componentes, de acordo com suas similaridades em torno das variáveis utilizadas (Ter Braak, 1986). As variáveis foram padronizadas para reduzir efeitos de escala. A ACP transforma um conjunto original de variáveis em outro conjunto de dimensão equivalente. Assim, as amostras (plantações) e as variáveis (atributos microbianos, $\mathrm{C}$ e $\mathrm{N}$ do solo e da serapilheira e atributos de qualidade da serapilheira) foram transformadas em coordenadas que correspondem à sua projeção nos eixos de ordenação, ou autovetores, representando o peso de cada variável sobre cada componente (eixo) e funcionando como coeficiente de correlação, que varia de -1 até +1 . As variáveis com elevado autovetor no primeiro eixo tendem a ter autovetor inferior no segundo eixo. Neste trabalho, considerou-se o autovetor $<0,7$ de baixa associação para a interpretação dos componentes principais (Wick et al., 1998).

Além disso, os componentes principais (eixos) são independentes entre si e estimados com o propósito de reter, em ordem de estimação, o máximo de informação em termos de variação total contida nos dados iniciais. O número de componentes utilizados para explicar a variação dos dados pode igualar-se ao número de variáveis, porém os eixos posteriores contribuem, cada vez menos, para explicar os dados (Cruz \& Regazzi, 1997). Na maioria dos estudos, como no caso deste trabalho, apenas os dois primeiros componentes são utilizados, considerados suficientes para explicar os dados pois, facilitam a interpretação do gráfico em duas dimensões (Gomes et al., 2004).

Um ponto qualquer plotado no diagrama (representando uma plantação de eucalipto) pode ser relacionado com cada seta (representando o atributo do solo ou serapilheira) por meio de perpendicularidade partindo da linha da seta até o referido ponto. A ordem na qual os pontos se projetam na seta, da sua extremidade até sua origem, dá uma indicação dessa relação. Plantações com sua projeção perpendicular próxima ou além da ponta da seta são mais positivamente correlacionadas e influenciadas pelo atributo em questão. Aquelas na extremidade oposta são influenciadas em menor grau. O ângulo de inclinação de cada seta com relação a cada eixo indica quão estreitamente correlacionado está o atributo com este eixo (Alvarenga \& Davide, 1999).

\section{RESULTADOS E DISCUSSÃO}

\section{Solo}

Os solos coletados neste trabalho são de elevada acidez e média fertilidade. Os teores de $\mathrm{C}$ orgânico e $\mathrm{N}$ total pouco variaram, e o teor de argila foi semelhante entre as plantações de eucalipto (Quadros 2 e 3). Gama-Rodrigues et al. (2005), em solos sob eucalipto com sete anos de idade e com baixo teor de argila, encontraram valores baixos de C orgânico $\left(7,30 \mathrm{~g} \mathrm{~kg}^{-1}\right)$ e $\mathrm{N}$ total $\left(0,40 \mathrm{~g} \mathrm{~kg}^{-1}\right)$, inferiores aos 
Quadro 3. Carbono orgânico, nitrogênio total, carbono e nitrogênio da biomassa microbiana e respiração acumulada de solo e serapilheira de plantações de eucalipto

\begin{tabular}{|c|c|c|c|c|c|c|c|c|c|}
\hline Plantação & $\mathbf{C}^{(1)}$ & $\mathbf{N}$ & CBM & NBM & $\mathbf{R A}$ & C:N microbiana & CBM:C & NBM:N & $\mathrm{qCO}_{2}$ \\
\hline \multirow[t]{2}{*}{ ano } & $\ldots \mathrm{g} \mathrm{kg}$ & 1 & 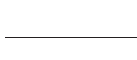 & $\mu \mathrm{g} \mathrm{g}{ }^{-1}$ & - & & — & 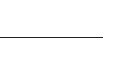 & $\mathrm{mg} \mathrm{g}^{-1} \mathrm{dia}^{-1}$ \\
\hline & \multicolumn{9}{|c|}{ Solo } \\
\hline 1 & $21,41 \mathrm{c}^{(2)}$ & $1,49 b$ & $143,71 \mathrm{ab}$ & $60,50 \mathrm{ab}$ & $15,20 \mathrm{~b}$ & $3,91 \mathrm{a}$ & $0,67 \mathrm{a}$ & $4,15 \mathrm{a}$ & $15,69 b$ \\
\hline 3 & $29,36 \mathrm{a}$ & $1,90 \mathrm{a}$ & $186,70 \mathrm{a}$ & $74,29 \mathrm{a}$ & $37,68 \mathrm{~b}$ & $3,00 \mathrm{a}$ & $0,63 \mathrm{a}$ & $4,09 \mathrm{a}$ & $20,82 b$ \\
\hline 5 & $27,00 \mathrm{ab}$ & $1,75 \mathrm{a}$ & $87,73 \mathrm{~b}$ & $43,84 \mathrm{~b}$ & $69,36 \mathrm{a}$ & $2,13 \mathrm{a}$ & $0,34 \mathrm{~b}$ & $2,50 \mathrm{~b}$ & $78,92 \mathrm{a}$ \\
\hline \multirow[t]{2}{*}{13} & $24,23 \mathrm{bc}$ & $1,85 \mathrm{a}$ & $180,46 a$ & $70,22 \mathrm{a}$ & $64,44 \mathrm{a}$ & $2,70 \mathrm{a}$ & $0,76 \mathrm{a}$ & $3,77 \mathrm{a}$ & $47,31 \mathrm{ab}$ \\
\hline & \multicolumn{9}{|c|}{ Serapilheira } \\
\hline 1 & $44,05 \mathrm{ab}$ & $13,03 \mathrm{a}$ & $7.589 \mathrm{a}$ & $3.924 \mathrm{a}$ & $8.498 \mathrm{a}$ & $2,09 \mathrm{a}$ & $17,26 \mathrm{a}$ & $35,76 \mathrm{a}$ & $147,42 \mathrm{a}$ \\
\hline 3 & $48,30 \mathrm{a}$ & $8,84 \mathrm{bc}$ & $2.181 \mathrm{~b}$ & $1.509 \mathrm{~b}$ & $3.121 \mathrm{~b}$ & $1,59 \mathrm{a}$ & $4,59 \mathrm{~b}$ & $17,21 \mathrm{bc}$ & $167,20 \mathrm{a}$ \\
\hline 5 & $40,40 \mathrm{~b}$ & $6,69 \mathrm{c}$ & $2.877 \mathrm{~b}$ & $1.687 \mathrm{~b}$ & $3.534 \mathrm{~b}$ & $1,76 \mathrm{a}$ & $7,65 \mathrm{~b}$ & $26,10 \mathrm{ab}$ & $133,47 \mathrm{a}$ \\
\hline 13 & $42,26 \mathrm{~b}$ & $9,80 \mathrm{~b}$ & $1.991 \mathrm{~b}$ & $1.037 \mathrm{~b}$ & $2.964 \mathrm{~b}$ & $2,41 \mathrm{a}$ & $4,81 \mathrm{~b}$ & $10,59 \mathrm{c}$ & $161,05 \mathrm{a}$ \\
\hline
\end{tabular}

(1) $\mathrm{C}-\mathrm{C}$ orgânico; $\mathrm{N}$ - N total; CBM - C da biomassa microbiana; NBM - N da biomassa microbiana; RA - Respiração acumulada; C:N microbiana- relação $\mathrm{C}$ da biomassa microbiana: $\mathrm{N}$ da biomassa microbiana; CBM:C - relação C da biomassa microbiana: $\mathrm{C}$ orgânico; NBM: $\mathrm{N}$ - relação $\mathrm{N}$ da biomassa microbiana: $\mathrm{N}$ total; $\mathrm{qCO}_{2}$ - Quociente metabólico. ${ }^{(2)}$ Médias seguidas da mesma letra, na coluna, não diferem entre si pelo teste de Duncan a $5 \%$.

observados neste trabalho. Já Mendham et al. (2002) encontraram valores médios de $\mathrm{C}$ e $\mathrm{N}$ de $39 \mathrm{e} 1,73 \mathrm{~g} \mathrm{~kg}^{-1}$, respectivamente, em solos com baixo teor de argila e baixo nível de fertilidade sob plantações de eucalipto.

Os resultados de C (CBM) e N (NBM) da biomassa microbiana variaram significativamente entre as plantações (idades) (Quadro 3). Os resultados estão de acordo com os dados reportados por Gama-Rodrigues et al. (2005), que, estudando plantações de eucalipto com sete anos de idade, observaram valores médios de CBM e NBM de 155,7 e 41,1 $\mu \mathrm{g} \mathrm{g}^{-1}$ de solo, respectivamente.

A respiração acumulada do solo (RA) variou de 15,2 a $69,4 \mu \mathrm{g} \mathrm{g}^{-1}$ com maiores valores verificados nas plantações com idades de cinco e 13 anos (Quadro 3). Gama-Rodrigues et al. (2005), em solos sob eucalipto, e Paulino (2003), em solo degradado também cultivados com essa essência, encontraram médias de RA de 45,2 e $26 \mu \mathrm{g} \mathrm{g}^{-1}$, respectivamente.

Em geral, houve pequena variação dos índices microbianos entre as idades. A relação C:N microbiana média foi de 2,9, valor inferior aos encontrados por Mendhan et al. (2002) em solo argiloso sob eucalipto com um e cinco anos de idade. As relações CBM:C e NBM:N médias foram 0,6 e 3,6 \%, respectivamente (Quadro 3). Gama-Rodrigues et al. (2005) encontraram, em solos sob eucalipto com sete anos de idade, relação CBM:C média de 2,2 \%. Monteiro (2001), também em solos cultivados com eucalipto, encontrou relação NBM:N média de $3,2 \%$. $\mathrm{O} \mathrm{qCO}_{2}$ (quociente metabólico) foi a variável que se destacou pela maior diferença entre o menor e o maior valor encontrado (80 \%) (Quadro 3). Esta variável apresentou média de $40,7 \mathrm{mg} \mathrm{g}^{-1}$ dia $^{-1}$ de C- $\mathrm{CO}_{2}$, próximo ao valor encontrado por Gama-Rodrigues et al. (2005).
A análise de componentes principais (ACP) para os atributos microbianos (CBM, NBM e RA) e químicos (C e N) do solo (Figura 1), apresentou autovalores de 49,5\% para o primeiro eixo (horizontal, CP1) e $42,7 \%$ para o segundo eixo (vertical, CP2), o que representa $92,2 \%$ da variância total acumulada nos dois primeiros eixos.

Os atributos do solo mais fortemente correlacionados com o primeiro eixo de ordenação foram $\mathrm{N}$ e $\mathrm{C}$, com valores de correlação de-1,0 e -0,84, respectivamente, caracterizando uma estreita associação entre esses elementos. Os atributos que apresentaram menor correlação com esse eixo foram RA $(-0,62), \operatorname{CBM}(-0,38)$ e NBM $(-0,38)$, tendo, portanto, menor importância para explicar este componente principal. O segundo eixo teve como atributos mais relevantes o CBM $(-0,92)$ e NBM $(-0,92)$, caracterizando o componente microbiano. A RA $(-0,61)$ também apresentou baixa correlação com este eixo. Salamanca et al. (2002), trabalhando em solos de floresta tropical secundária nas Filipinas, observaram que os conteúdos de adenosina trifosfato (ATP), NBM, RA, ergosterol (medida indireta de atividade fúngica) e CBM foram os atributos de maior correlação com o primeiro eixo e o consideraram o eixo que representou o status microbiológico do solo. Dinesh et al. (2003), também utilizando a análise de componentes principais para interpretar a relação entre atributos químicos e microbianos de solos sob diferentes coberturas florestais, verificaram no primeiro eixo maiores correlações entre CBM, NBM, RA, ergosterol, ATP, C e N, e sugeriram que a associação entre essas variáveis caracterizaria a atividade microbiana e a dependência dos microrganismos do solo pelos teores de matéria orgânica e N total. 


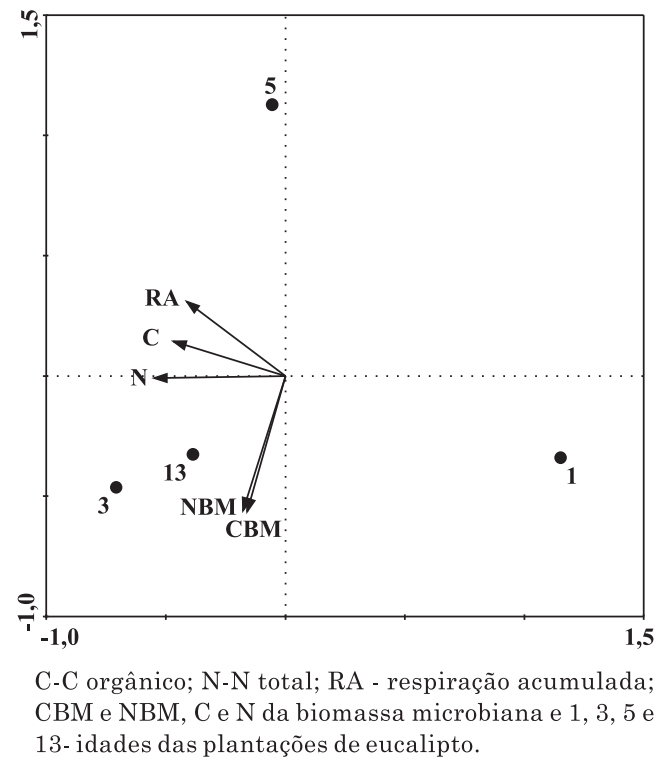

Figura 1. Diagrama de ordenação produzido pela análise de componentes principais dos atributos do solo.

Os teores de $\mathrm{C}$ orgânico e $\mathrm{N}$ total foram as variáveis mais discriminantes na avaliação da variação total entre os solos sob eucalipto de diferentes idades. Esse resultado contraria comprovações de que a biomassa microbiana é um indicador mais sensível de mudanças nos teores de MOS do que o teor de C orgânico do solo (Powlson et al., 1987; Anderson \& Domsch, 1989; Sparling, 1992, Gama-Rodrigues, 1999).

A dispersão gráfica das plantações em relação aos dois eixos demonstra que houve dissimilaridade entre as idades (Figura 1). O eucalipto de um ano localizarase à direita do diagrama, próxima de CP1, enquanto os das demais idades agruparam-se à esquerda. Estes resultados são decorrentes dos menores valores de $\mathrm{C}$ orgânico e $\mathrm{N}$ total observados no eucalipto de um ano, tendo em vista que estas variáveis foram as mais importantes para a explicação da variância total dos resultados.

O posicionamento das plantações de 3, 5 e 13 anos à esquerda do gráfico, próximas de CP2, demonstra que CBM e NBM, atributos mais correlacionados com o segundo eixo, foram as características de maior influência para a diferenciação dessas plantações. O fato do eucalipto de cinco anos estar localizado no quadrante superior e os de 3 e 13 anos no quadrante inferior indica maior similaridade entre estas duas últimas idades. Tal semelhança está relacionada aos maiores valores de CBM e NBM observado nessas idades, enquanto os de cinco anos apresentaram os menores valores desses atributos. Observaram-se as menores relações CBM:C e NBM:N e o maior $\mathrm{qCO}_{2}$ (Quadro 3) na plantação de cinco anos, sugerindo menor eficiência da biomassa microbiana na imobilização de $\mathrm{C}$ e $\mathrm{N}$ nessa idade. Contudo, as variáveis medidas neste trabalho não explicam esta redução do $\mathrm{C}$ e $\mathrm{N}$ microbiano aos cinco anos, uma vez que os atributos de qualidade da serapilheira não variaram entre as idades. Monteiro (2001) encontrou correlações negativas e significativas entre as relações lignina:N e polifenol:N com CBM e NBM de solos sob floresta natural e eucalipto, sugerindo que a eficiência da biomassa microbiana na utilização do $\mathrm{C}$ e $\mathrm{N}$ acumulados estaria diretamente relacionada à qualidade da serapilheira depositada.

\section{Serapilheira}

Houve pequena variação do teor de C orgânico entre as idades. Os maiores valores foram observados nas idades de um e três anos (Quadro 3). O N total apresentou maior amplitude de variação, sendo significativamente superior na idade de um ano (Quadro 3). Ndaw (2003) encontrou na serapilheira de eucalipto, na região norte fluminense, teores de $\mathrm{C}$ e $\mathrm{N}$ de 38 e $7 \mathrm{~g} \mathrm{~kg}^{-1}$, respectivamente.

O CBM, o NBM e a RA foram significativamente maiores na idade de um ano. A média geral desses atributos foram $3.659,4 \mathrm{mg} \mathrm{g}^{-1}$ de C, 2.039,5 mg g-1 de $\mathrm{N}$ e $4.529,31 \mathrm{mg} \mathrm{g}^{-1}$ de $\mathrm{C}-\mathrm{CO}_{2}$, respectivamente (Quadro 3). Em sítios de eucalipto em diferentes regiões do Brasil, Gama-Rodrigues (1997a) verificou CBM de $2.902 \mathrm{mg} \mathrm{g}^{-1}$ de $\mathrm{C}$ e de NBM de $1.068 \mathrm{mg} \mathrm{g}^{-1}$ de $\mathrm{N}$ de serapilheira, e Monteiro (2001) encontrou RA de $5.750 \mathrm{mg} \mathrm{g}^{-1}$. A relação $\mathrm{C}: \mathrm{N}$ microbiana e o $\mathrm{qCO}_{2}$ não apresentaram variação significativa entre as idades (Quadro 3). Entretanto, as relações CBM:C e NBM:N foram significativamente maiores na plantação de eucalipto com idade de um ano.

Os teores de celulose (CEL) e polifenóis (POL) e a relação POL:N não diferiram estatisticamente entre as idades das plantações (Quadro 4). O teor de lignina (LIG), porém, foi significativamente menor na idade de um ano, que também apresentou relações CEL:N e LIG:N estatisticamente inferiores às das demais idades, as quais não diferiram entre si. Monteiro (2001) encontrou na serapilheira de eucalipto de 20 anos teores médios de CEL, LIG e POL bem próximos e valores das relações LIG:N e POL:N superiores aos observados neste estudo.

Para os atributos da serapilheira (C, N, atributos microbianos e composição orgânica), a ACP (Figura 2) apresentou autovalores de $73,8 \%$ para o primeiro eixo e de $20,5 \%$ para o segundo eixo, o que representa $94,4 \%$ da variância total acumulada nos dois primeiros eixos, com maior percentagem da variação explicada no primeiro eixo.

Os atributos da serapilheira mais correlacionados com o primeiro eixo foram NBM $(1,0), \operatorname{CBM}(0,99)$, RA (0,99), CEL (-0,99), LIG $(-0,97)$ e N $(0,74)$, caracterizando a estreita relação entre os processos de imobilização e mineralização de $\mathrm{C}$ e $\mathrm{N}$, exercidos pela biomassa microbiana, e a qualidade orgânica e nutricional do material depositado. No segundo eixo, 
Quadro 4. Fitomassa, teores de celulose, lignina e polifenóis, e relações celulose:N, lignina: $\mathrm{N}$ e polifenóis: $\mathrm{N}$ da serapilheira de plantações de eucalipto

\begin{tabular}{|c|c|c|c|c|c|c|c|}
\hline Plantação & Fitomassa & CEL $^{(1)}$ & POL & LIG & POL:N & CEL:N & LIG:N \\
\hline ano & $M g h a^{-1^{1}}$ & - & $-\mathrm{g} \mathrm{kg}^{-1}$ & - & 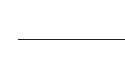 & $\%$ & 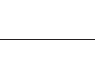 \\
\hline 1 & $1,56 \mathrm{~d}^{(2)}$ & $120,0 \mathrm{a}$ & $81,1 \mathrm{a}$ & $112,0 \mathrm{c}$ & $8,2 \mathrm{a}$ & $12,9 \mathrm{~b}$ & $6,3 \mathrm{~b}$ \\
\hline 3 & $3,84 \mathrm{c}$ & $250,0 \mathrm{a}$ & $128,0 \mathrm{a}$ & $270,0 \mathrm{a}$ & $13,9 \mathrm{a}$ & $27,2 \mathrm{a}$ & $29,3 \mathrm{a}$ \\
\hline 5 & $15,25 \mathrm{~b}$ & $244,4 \mathrm{a}$ & $89,5 \mathrm{a}$ & $214,0 \mathrm{~b}$ & $11,0 \mathrm{a}$ & $30,0 \mathrm{a}$ & $26,9 \mathrm{a}$ \\
\hline 13 & $22,86 \mathrm{a}$ & $262,0 \mathrm{a}$ & $99,8 \mathrm{a}$ & $302,0 \mathrm{a}$ & $10,2 \mathrm{a}$ & $26,8 \mathrm{a}$ & $30,9 \mathrm{a}$ \\
\hline
\end{tabular}

(1) CEL - Celulose; POL - Polifenóis; LIG - Lignina; POL:N - relação polifenóis: N total; CEL:N - relação celulose: N total; LIG:N - relação lignina: $\mathrm{N}$ total. ${ }^{(2)}$ Médias seguidas da mesma letra, na coluna, não diferem entre si pelo teste de Duncan, a $5 \%$.

os teores de $\mathrm{N}$ total $(-0,67), \mathrm{C}$ orgânico $(-0,58)$ e polifenóis $(-0,34)$ apresentaram baixa correlação. Dessa forma, os atributos microbianos e os teores de celulose, lignina e $\mathrm{N}$ da serapilheira foram as variáveis que melhor explicaram a variação total dos dados, o que reforçaria a inter-relação entre esses atributos. Este resultado é corroborado por Monteiro \& GamaRodrigues (2004), que encontraram correlações significativas entre atributos microbianos e índices de qualidade da serapilheira de uma floresta natural.

Quanto à distribuição dos povoamentos de eucalipto apresentada no diagrama de ordenação da ACP (Figura 2), a localização da plantação de um ano à direita, próximos de CP1, e o agrupamento dos demais à esquerda, semelhante ao observado para o solo, demonstram que houve dissimilaridade entre plantações com idade de um ano em relação àquelas com três, cinco e 13 anos. Esses resultados são decorrentes dos maiores valores de CBM, NBM, RA e $\mathrm{N}$, e menores valores de CEL e LIG observados na plantação de um ano, uma vez que essas variáveis foram as mais importantes para a explicação da variância total das plantações de eucalipto. Isto sugere que a biomassa microbiana passou a ser menos ativa e, ou, menos eficiente com o aumento da idade. Esses resultados são corroborados pelo teor de LIG (Quadro 4) e pelas relações CBM:C, NBM:N (Quadro 3) e LIG:N (Quadro 4), que indicam que, na plantação com um ano, o C orgânico e $\mathrm{N}$ total da serapilheira do eucalipto apresentaram melhor qualidade quando comparados com os das demais idades. Dessa forma, a diminuição do teor de $\mathrm{N}$ e o aumento das relações CEL:N e LIG:N nos plantios mais antigos conferiram maior nível de recalcitrância à serapilheira e, conseqüentemente, menor acesso dos microrganismos ao substrato, limitando a atividade $\mathrm{e}$ o crescimento da biomassa microbiana.

A distribuição das plantações de eucalipto no diagrama da figura 2 , portanto, reflete o efeito da idade na acumulação e a qualidade da serapilheira (Quadro 4), demonstrando a diferenciação das idades avançadas em relação à fase inicial de estabelecimento da cultura (um ano).

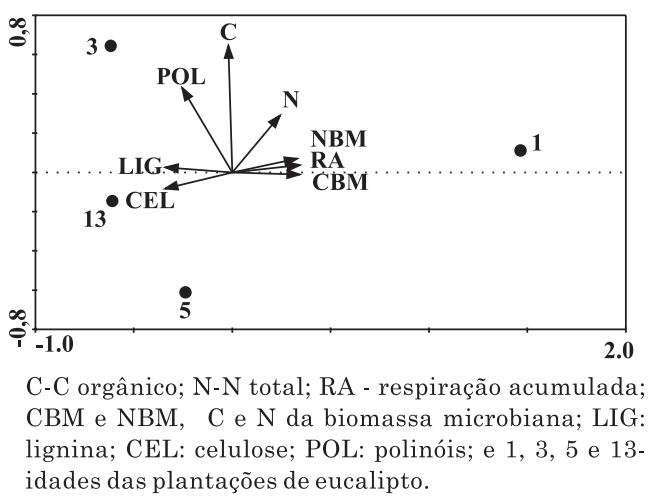

Figura 2. Diagrama de ordenação produzido pela análise de componentes principais dos atributos da serapilheira.

Os maiores teores de $\mathrm{C}$ e $\mathrm{N}$ microbiano da serapilheira em relação aos encontrados no solo (Quadro 3) são corroborados por resultados obtidos por Gama-Rodrigues et al. (2005), que sugeriram que a serapilheira de povoamentos de eucalipto, em diferentes sítios florestais, seria importante reserva de $\mathrm{C}$ e $\mathrm{N}$ microbiano. Além disso, como a biomassa microbiana constitui parte do $\mathrm{N}$ potencialmente mineralizável (Marumoto et al., 1982; Bonde et al., 1988), a quantidade de $\mathrm{N}$ acumulado na biomassa microbiana da serapilheira teria relevante contribuição no suprimento de $\mathrm{N}$ em plantações de eucalipto, uma vez que representa grande reservatório de $\mathrm{N}$ a ser liberado ao longo do tempo por meio da ciclagem de nutrientes.

\section{CONCLUSÕES}

1. No solo, os teores de $\mathrm{C}$ orgânico e $\mathrm{N}$ total foram indicadores mais discriminantes que a biomassa microbiana para aferir variações entre as plantações (idades) de eucalipto. Na serapilheira, ao contrário, tanto os atributos microbianos quanto os teores de celulose, lignina e $\mathrm{N}$ foram relevantes na discriminação das diferentes idades do eucalipto. 
2. A qualidade orgânica da serapilheira influenciou diretamente seus atributos microbianos.

\section{LITERATURA CITADA}

ALVARENGA, M.I.N. \& DAVIDE, A.C. Características físicas e químicas de um Latossolo Vermelho-Escuro e a sustentabilidade de agroecossistemas. R. Bras. Ci. Solo, 23:933-942, 1999.

ANDERSON, J.D. \& INGRAM, J.S.I. Tropical soil biology and fertility: A handbook of methods. 2.ed. Wallingford, CAB International, 1996. 171p.

ANDERSON, T.H. \& DOMSCH, K.H. Ratios of microbial biomass carbon to total organic carbon in arable soils. Soil Biol. Biochem., 21:471-479, 1989.

ANDERSON, T.H. \& DOMSCH, K.H. The metabolic quotient $\left(\mathrm{qCO}_{2}\right)$ as a specific activity parameter to assess the effects of environmental conditions, such as $\mathrm{pH}$, on the microbial biomass of forest soils. Soil Biol. Biochem., 25:393-395, 1993.

BARRETO, P.A.B. Biomassa microbiana e mineralização de carbono e nitrogênio em povoamentos de eucalipto, em uma seqüência de idades. Campos dos Goytacazes, Universidade Estadual do Norte Fluminense, 2004. 86p. (Tese de Mestrado)

BARROS, N.F. \& COMERFORD, N.B. Sustentabilidade da produção de florestas plantadas na região tropical. In: ALVAREZ V., V.H.; SCHAEFER, C.E.G.R.; BARROS, N.F.; MELLO, J.W.V. \& COSTA, L.M., eds Tópicos em ciência do solo. Viçosa, MG, Sociedade Brasileira de Ciência do Solo, 2002. v.2. p.487-592.

BARROS, N.F.; NEVES, J.C.L. \& NOVAIS, R.F. Fertilização e correção do solo para o plantio de eucalipto. In: BARROS, N.F. \& NOVAIS, R.F., eds. Relação solo-eucalipto. Viçosa, MG, Folha de Viçosa, 1990. p.127-186.

BARROS, N.F.; NEVES, J.C.L. \& NOVAIS, R.F. Mineral fertilizer recommendations for eucalypt plantations. In: GONÇALVES, J.L.M. \& BENEDETTI, V., orgs. Forest nutrition and fertilization. Piracicaba, IPEF, 2004. p.269305.

BATAGLIA, O.C.; FURLANI, A.M.C.; TEIXEIRA, J.P.F.; FURLANI, P.R. \& GALLO, J.R. Métodos de análise química de plantas. Campinas, Instituto Agronômico, 1983. 48p. (Boletim Técnico, 78)

BONDE, T.A.; SCHNÜRER, J. \& ROSSWALL, T. Microbial biomass as a fraction of potentially mineralizable nitrogen in soils from long-term field experiments. Soil Biol. Biochem., 20:447-452, 1988.

CRUZ, C.D. \& REGAZZI, A.J. Modelos biométricos aplicados ao melhoramento genético. 2.ed. Viçosa, MG, Universidade Federal de Viçosa, 1997. 390p.

D'ANDREA, A.F.; SILVA, M.L.N.; CURI, N.; SIQUEIRA, J.O. \& CARNEIRO, M.A.C. Atributos biológicos da qualidade do solo em sistemas de manejo na região do Cerrado no Sul do Estado de Goiás. R. Bras. Ci. Solo, 26:913-923, 2002 .
DEFELIPO, B.V. \& RIBEIRO, A.C. Análise química do solo. Viçosa, MG, Universidade Federal de Viçosa, 1981. 17p. (Boletim de Extensão, 29)

DINESH, R.; GHOSHAL CHAUDHURI, S.; GANESHAMURTHY, A.N. \& CHANCHAL DEY. Changes in soil microbial indices and their relationships following deforestation and cultivation in wet tropical forests. Soil Biol. Biochem., 24:17-26, 2003.

EMPRESA BRASILEIRA DE PESQUISA AGROPECUÁRIA EMBRAPA. Serviço Nacional de Levantamento e Conservação de Solos. Manual de métodos de análise de solo. 2.ed. Rio de Janeiro, 1997. 412p.

GAMA-RODRIGUES, A.C. Ciclagem de nutrientes por espécies florestais em povoamentos puros e mistos em solos de tabuleiro na Bahia, Brasil. Viçosa, MG, Universidade Federal de Viçosa, 1997b. 107p. (Tese de Doutorado)

GAMA-RODRIGUES, E.F. Biomassa microbiana e ciclagem de nutrientes. In: SANTOS, G.A. \& CAMARGO, F.A.O., eds. Fundamentos da matéria orgânica do solo: Ecossistemas tropicais e subtropicais. Porto Alegre, Gênesis, 1999. p.227-243.

GAMA-RODRIGUES, E.F. Carbono e nitrogênio da biomassa microbiana da serapilheira de povoamentos de eucalipto. Seropédica, Universidade Federal Rural do Rio de Janeiro, 1997a. 108p. (Tese de Doutorado)

GAMA-RODRIGUES, E.F.; BARROS, N.F.; GAMARODRIGUES, A.C. \& SANTOS, G.A. Nitrogênio, carbono e atividade da biomassa microbiana do solo em plantações de eucalipto. R. Bras. Ci. Solo, 29:393-901, 2005.

GAMA-RODRIGUES, E.F.; GAMA-RODRIGUES, A.C. \& BARROS, N.F. Biomassa microbiana de carbono e de nitrogênio de solos sob diferentes coberturas vegetais. R. Bras. Ci. Solo, 21:361-365, 1997.

GOMES, J.B.V.; CURI, N.; MOTTA, P.E.F.; KER, J.C.; MARQUES, J.J.G.S.M. \& SCHULZE, D.G. Análise de componentes principais de atributos físicos, químicos e mineralógicos de solos do bioma Cerrado. R. Bras.Ci. Solo, 28:137-154, 2004.

GONÇALVES, J.L.M.; MENDES, K.C.F.S. \& SASAKI, C.M. Mineralização de nitrogênio e carbono em ecossistemas florestais naturais e implantados no Estado de São Paulo. R. Bras. Ci. Solo, 25:601-616, 2001.

GONÇALVES, J.L.M.; STAPE, J.L.; BENEDETT, V.; FESSEL, V.A.G. \& GAVA, J.L. Reflexos do cultivo mínimo e intensivo do solo em sua fertilidade e na nutrição das árvores. In: GONÇALVES, J.L.M. \& BENEDETT, V., eds. Nutrição e fertilização florestal. Piracicaba, IPEF, 2000. p.1-58.

GRISI, B.M. Biomassa e a atividade de microrganismos do solo: Revisão metodológica. R. Nordestina Biol., 10:1-22, 1995.

JENKINSON, D.S. \& POWLSON, D.S. The effects of biocidal treatments on metabolism in soil. V. A method for measuring soil biomass. Soil Biol. Biochem., 8:209-213, 1976.

JOERGENSEN, R.G. \& BROOKES, P.C. Ninhydrin-reactive nitrogen measurements of microbial biomass in $0,5 \mathrm{M}$ $\mathrm{K}_{2} \mathrm{SO}_{4}$ soil extracts. Soil Biol. Biochem., 22:1033-1027, 1990. 
MARUMOTO, T.; ANDERSON, J.P.E. \& DOMSCH, K.H. Mineralization of nutrients from soil microbial biomass. Soil Biol. Biochem., 14:469-475, 1982.

MENDHAM, D.S.; SANKARAN, K.V.; O'CONNELL, A.M. \& GROVE, T.S. Eucalyptus globulus harvest residue management effects on soil carbon and microbial biomass at 1 and 5 years after plantation establishment. Soil Biol. Biochem., 34:1903-1912, 2002.

MONTEIRO, M. T. Carbono, nitrogênio e atividade da biomassa microbiana: Indicadores da qualidade do solo e da serapilheira em sítios florestais do Norte Fluminense. Campos dos Goytacazes, Universidade Estadual do Norte Fluminense, 2001. 75p. (Tese de Mestrado)

MONTEIRO, M.T. \& GAMA-RODRIGUES, E.F. Carbono, nitrogênio e atividade da biomassa microbiana em diferentes estruturas de serapilheira de uma floresta natural. R. Bras. Ci. Solo, 28:819-826, 2004.

NDAW, S.M. Diversidade, biomassa e atividade microbiana como indicadores da qualidade do solo e da serapilheira em sítios sob diferents coberturas vegetais na região Norte Fluminense. Campos dos Goytacazes, Universidade Estadual do Norte Fluminense, 2003. 98p. (Tese de Mestrado)

PAULINO, G.M. Cobertura florestal e qualidade de solos em terras degradadas no Norte Fluminense. Campos dos Goytacazes, Universidade Estadual do Norte Fluminense, 2003. 67p. (Tese de Mestrado)

POGGIANI, F.; COUTO, H.T.Z.; CORRADINI, L. \& FAZZIO, E.C.M. Exportação de biomassa e nutrientes através da exportação dos troncos e das copas de um povoamento de Eucalyptus saligna. R. IPEF, 25:37-39, 1983.

POWLSON, D.S.; BROOKES, P.C. \& CHRISTENSEN, B.T. Measurement of soil microbial biomass provides an early indication of changes in the total soil organic matter due to straw incorporation. Soil Biol. Biochem., 19:159-164, 1987.

REIS, M.G.F.; BARROS, N.F. \& KIMMINS, J.P. Acúmulo de nutrientes em uma seqüência de idade de Eucalyptus grandis W. Hill (ex-maiden) plantado no Cerrado em duas áreas com diferentes produtividades, em Minas Gerais. R. Árvore, 11:1-15, 1987.
SALAMANCA, E.F.; RAUBUCH, M. \& JOERGENSEN, R.G. Relationships between soil microbial indices in secondary tropical forest soils. Soil Biol. Biochem., 21:211-219, 2002.

SCHUMACHER, M.V. \& POGGIANI, F. Produção de biomassa e remoção de nutrientes em povoamentos de Eucalyptus camaldulensis Dehnh, Eucalyptus grandis Hill ex Maiden e Eucalyptus torelliana F. Muell, plantados em Anhembí, SP. Ci. Flor., 1:21-34, 1993.

SMITH, J.L. \& PAUL, E.A. The significance of soil microbial biomass estimations. In: BOLLAG, J.M. \& STOTZKY, G., eds. Soil Biochemistry. New York, Marcel Dekker, 1990. p.357-396

SPARLING, G.P. Ratio of microbial biomass carbon to soil organic carbon as a sensitive indicator of changes in soil organic matter. Aust. J. Soil Res., 30:195-207, 1992.

TATE, K.R.; ROSS, D.J. \& FELTHAM, C.W. A direct extraction method to estimative soil microbial C: Effects of experimental variables and some different calibration procedures. Soil Biol. Biochem., 20:329-335, 1988.

TER BRAAK, C.J.F. Canonical correspondence analysis: A new eingvector technique for multivariate direct gradient analysis. Ecology, 67:1167-1179, 1986

VAN SOEST, P. \& WINE, R.H. Development of a comphrehensive system of feed analysis and its applications to forages. J. Assoc. Official Agr. Chem., 51:780-785, 1968

VARGAS, L.K. \& SCHOLES, D. Nitrogênio da biomassa microbiana, em solo sob diferentes sistemas de manejo, estimado por métodos de fumigação. R. Bras. Ci. Solo, 22:411-417, 1998.

WARDLE, D.A.A. comparative assessment of factors which influence microbial biomass carbon and nitrogen levels in soil. Biol. Rev., 67:21-358, 1992.

WICK, B.; TIESSEN, H. \& MENEZES, R. Land use changes following the conversion of the natural vegetation into silvo-pastoral systems in semi-arid NE Brazil. Plant Soil, 222:59-70, 1998. 